\title{
Poles Extraction of Radar Target Based on Narrowband Frequency Response Approximation
}

\author{
Xuran Xing ${ }^{1, *}$, Hongzhong Zhao ${ }^{2}$, and Xin $\mathrm{Jia}^{3}$ \\ ${ }^{1}$ Sapace Engineering University, Beijing, 101416, China \\ ${ }^{2}$ Sapace Engineering University, Beijing, 101416, China \\ ${ }^{3}$ Sapace Engineering University, Beijing, China
}

\begin{abstract}
Pole is the most important feature of radar target in resonance region, and it is attitude insensitive. The poles extraction requires UWB (ultra-wideband) late-time response signal or frequency response data, which is difficult to achieve through general equipment. This paper proposed a method for extracting poles from narrowband data. At first, the frequency response equation of the resonance region was derived. Then, the equation parameters were solved by the interior point method through the narrowband data. At last, the zeros of the transfer function denominator were calculated. FEKO simulation data test verifies that the method has extrapolation ability.
\end{abstract}

\section{Introduction}

Radar target recognition technology determines the target properties and categories based on the target electromagnetic scattering properties. When the target size and the wavelength size are at the same order of magnitude, the target is in the resonant zone and the main electromagnetic scattering property is the pole. The pole is insensitive of the observation angle and the polarization of the radar. It is only related to the size, shape and material of the target itself, so it is a robust electromagnetic feature for target recognition.

One of the conditions for poles extraction is UWB signals, but most radar devices do not have the capability of ultra-wideband. Existing poles extraction algorithms, such as prony method[1], matrix pencil method[2] and iterative method[3], do not have the ability to extract poles from narrowband data. This paper proposed a poles extraction method from narrowband frequency response data. The transfer function equations of the resonance region was derived according to the singularity expansion method (SEM) theory. Then, the primal-dual interior point method solved the illconditioned equations and obtained the transfer function coefficients. The zeros of the transfer function denominator are the poles. FEKO calculated the far-field data of thin-cylinder, rectangle, and a complex target, and validated the algorithm.

\section{Electromagnetic characteristics of radar target in resonance region}

The singularity expansion method (SEM) was proposed when Baum studied the back response of radar echoes in
1971[4]. SEM describes the characteristics of the echo and transfer function in the resonance region systematically. The ultra-wideband pulse signal is usually used as the excitation when studying the echo signals in resonance region, so the response signal is very short in time domain and it is divided into two parts: the early-time response and the late-time response. The early-time response describes the scattering center characteristics of the target, varying with the observation angle; the late-time response describes the poles characteristics of the target, and the position of the poles does not change with the observation angle. The latetime response can be represented as follows:

$$
y(t)=\sum_{m}^{M} r_{m} e^{s_{m} t}
$$

where $r_{m}$ are the residues, $s_{m}=\sigma_{\mathrm{m}}+\omega_{\mathrm{m}} j$ are the poles. The real part $\sigma_{\mathrm{m}}$ is the attenuation factor and the imaginary part $\omega_{\mathrm{m}}$ is the angular frequency. The residues change when changing the observation angle, but the poles remain stable. The transfer function is modelled as the sum of a residue series plus and an entire function:

$$
H(s)=\sum_{i=1}^{M} \frac{r_{i}}{s-s_{i}}+C(s)
$$

In time domain, the residue series plus corresponds to late-time response, and the entire functions corresponds to early-time response.

\footnotetext{
*Corresponding author: xxr01839@mail.ustc.edu.cn
} 
There are two ways to acquire the late-time response signal. One is exciting target by UWB pulse signal in time domain, this method requires wide bandwidth and high sampling rate of equipment. The other way is transmitting a series of simple-frequency signal and calculating the frequency response value at each frequency point. Then, the late-time signal was reconstructed through inverse Fourier transform to the frequency response. The second methods are easier to implement and have greater application value. Existing poles extraction algorithms rely on the late-time signals, so UWB frequency response data is necessary. When the UWB condition is not satisfied, even if the data near the poles are acquired, the poles can not be accurately estimated. This constraint limits the application of poles as a target identification method.

\section{Poles extraction of radar target under narrow-band conditions}

\subsection{Transfer function equation}

The entire functions is complex and its energy is small, so it is usually neglected when we concern about the poles. Formula 2 is simplified as:

$$
H(s)=\sum_{i=1}^{M} \frac{r_{i}}{s-s_{i}}=\frac{\sum_{i=0}^{M-1} b_{i} s^{i}}{1+\sum_{i=1}^{M} a_{i} s^{i}}
$$

Multiplying both sides by the denominator and transposition

$$
H(s) \times \sum_{i=1}^{M} a_{i} s^{i}-\sum_{i=0}^{M-1} b_{i} s^{i}=-H(s)
$$

Discretization of formula 5 is expressed as below:

$$
\boldsymbol{A} \cdot \boldsymbol{x}=\boldsymbol{b}
$$

Where

$$
\begin{aligned}
& A=\left[\begin{array}{cccccccc}
H_{1} s_{1} & H_{1} s_{1}{ }^{2} & \cdots & H_{1} s_{1}{ }^{M} & -s_{1}^{0} & -s_{1}{ }^{1} & \cdots & -s_{1}{ }^{M-1} \\
H_{2} s_{2} & H_{2} s_{2}^{2} & \cdots & H_{2} s_{2}{ }^{M} & -s_{2}^{0} & -s_{2}{ }^{1} & \cdots & -s_{2}{ }^{M-1} \\
\vdots & \vdots & \ddots & \vdots & \vdots & \vdots & \ddots & \vdots \\
H_{N} s_{N} & H_{N} s_{N}{ }^{2} & \cdots & H_{N} s_{N}{ }^{M} & -s_{N}^{0} & -s_{N}{ }^{1} & \cdots & -s_{N}{ }^{M-1}
\end{array}\right], \\
& \boldsymbol{x}=\left[\begin{array}{llllllll}
a_{1} & a_{2} & \cdots & a_{M} & b_{0} & b_{1} & \cdots & b_{M-1}
\end{array}\right]^{T} \\
& \text { is the }
\end{aligned}
$$
and $\boldsymbol{b}=-H=-\left[H\left(j w_{1}\right) H\left(j w_{2}\right) \cdots H\left(j w_{N}\right)\right]^{T}$ is the response data collected in frequency domain. The parameterized transfer function is realized by solving the parameter vector $\boldsymbol{x}$.

\subsection{Solving the transfer function equation}

For the overdetermined equation of Formula 5, the usual solving method is calculating the least square solution $\boldsymbol{x}=\left(\boldsymbol{A}^{T} \boldsymbol{A}\right)^{-1} \boldsymbol{A}^{T} \boldsymbol{b}$. But this method is not applicable to ill conditioned equations. The problem of poles estimation is a ill conditioned problem[5].Ill conditioned means that the matrix $\boldsymbol{A}$ approach to singularity, at this point, the solution of the equation is unstable.

In order to obtain accurate parameters, the problem of parameter solving is considered as a nonlinear programming problem.

$$
\begin{aligned}
& \min \|\boldsymbol{A} \boldsymbol{x}-\boldsymbol{b}\|_{2} \\
& \text { s.t. } \quad \boldsymbol{A} \boldsymbol{x}=\boldsymbol{b}
\end{aligned}
$$

It is also a quadratic programming problem as follows

$$
\begin{aligned}
& \min \boldsymbol{x}^{T} \boldsymbol{Q x}-2 \boldsymbol{c}^{T} \boldsymbol{x} \\
& \text { s.t. } \quad \boldsymbol{A} \boldsymbol{x}=\boldsymbol{b}
\end{aligned}
$$

Where $\boldsymbol{Q}=\boldsymbol{A}^{T} \boldsymbol{A}$ and $c_{i}=b_{i} \sum_{j=1}^{2 M+1} a_{i j}$.

Define $\boldsymbol{X}=\boldsymbol{x} \cdot \boldsymbol{x}^{T}$, the quadratic programming problem has the following relaxation model [6]

$$
\begin{gathered}
\min \boldsymbol{Q} \cdot \boldsymbol{X} \\
\text { s.t. } \quad \boldsymbol{A x}=\boldsymbol{b} \\
\boldsymbol{X} \geq 0
\end{gathered}
$$

At this point, the problem has been transformed into a linear semidefinite programming problem. Semidefinite programming is an extension of programming problems. It is a new branch of mathematical programming developed in recent 20 years, with good mathematical properties and extensive engineering applications[7]. When the ill conditioned equations are transformed into linear semidefinite programming problems, some sophisticated optimization algorithms can be used to guarantee the accuracy and efficiency. This paper called the SDPT3, and used the primal-dual interior point method to solve above problem[8].

Interior point method constructs the objective function with penalty function, then the constrained optimization problem is transformed into an unconstrained optimization problem. The penalty function is small within the feasible region, and it is large at the boundary of the feasible region, so that the parameters in each iteration do not go beyond the feasible domain. The procedure of the primal-dual interior point method is described below.

For standard linear semidefinite programming problem as follows

$$
\begin{gathered}
\min \boldsymbol{C} \bullet \boldsymbol{X} \\
\boldsymbol{A} \bullet \boldsymbol{X}=b_{k}, k=1, \ldots, m \\
\boldsymbol{X} \geq 0
\end{gathered}
$$

and its dual problem

$$
\begin{gathered}
\max \boldsymbol{b}^{T} \boldsymbol{y} \\
\sum_{k=1}^{m} \boldsymbol{y} k \boldsymbol{A}_{k}+\boldsymbol{Z}=\boldsymbol{C} \\
\boldsymbol{Z} \geq 0
\end{gathered}
$$


The primal-dual interior point method can be summarized as following steps [8]:

1.Compute the duality gap $\mu=\boldsymbol{X} \cdot \boldsymbol{Z} / n$ and stop the iteration if it is smaller than the threshold;

2.Compute the search direction $(\Delta \boldsymbol{X}, \Delta \boldsymbol{y}, \Delta \boldsymbol{Z})$;

3.Update $(\boldsymbol{X}, \boldsymbol{y}, \boldsymbol{Z})$ to $(\boldsymbol{X}+\alpha \Delta \boldsymbol{X}, \boldsymbol{y}+\beta \Delta \boldsymbol{y}, \boldsymbol{Z}+\beta \Delta \boldsymbol{Z})$,

where

$$
\alpha=\min \left(1,-\frac{\gamma}{\lambda_{\min }\left(\boldsymbol{X}^{-1} \Delta \boldsymbol{X}\right)}\right)
$$
and

$\beta=\min \left(1,-\frac{\gamma}{\lambda_{\min }\left(\boldsymbol{Z}^{-1} \Delta \boldsymbol{Z}\right)}\right) ;$

4.Set step-length

parameter $\gamma=0.9+0.09 \min (\alpha, \beta)$ and return to step 1 .

\subsection{Model order determination}

The order of the transfer function is the number of poles, and it is unknown for the unknown target. In the case of narrowband data, the algorithm does not care about the number of all poles, because the purpose is to extract the poles near the narrow band. However, we are concerned with the effect of order on the fitting degree. If the order is too small, the transfer function model can not fit the collected narrowband data, and the poles can not be accurately extracted; if the order is too large, the ill conditioned is too serious, result in arithmetic overflow. In order to determine the proper order, the different order equations are constructed, and then the frequency response $H_{i}(j w)$ is reconstructed by the parameters respectively. Calculate the errors of the reconstructed responses between the original data as follows:

$$
\operatorname{error}(i)=\left\|H_{i}(j w)-H(j w)\right\|_{2}
$$

The order with the least error is considered as the order of the transfer function.

\subsection{Poles estimation}

The poles can be computed after obtain the parameterized transfer function. Calculate the zeros of the transfer function denominator as below

$$
a_{M+1} s^{M+1}+a_{M} s^{M}+\cdots+a_{1} s^{1}+1=0
$$

Since the equations are constructed by narrowband data, the solutions near the narrow band are accurate, while the solutions of other frequency bands are wrong, so the solutions must be cleaned up. Solutions both near the narrow band and in the second quadrant of the Laplace domain are preserved as the poles. To sum up, the algorithm proposed in this paper is summarized as follows:

(1) Set the model order $i=i_{0}$;

(2) Construct and solve the equation as formula (5);

(3) Reconstruct the frequency response by the parameters of step2, then calculate the error by formula (11);

(4) If the equation is overflow, proceed to next step, and if not, set $i=i+1$ and return to step 2 ;
(5) Compare the errors, the solution with the minimum error is chosen as the final parameters of the transfer function;

(6) Clear the result and preserve the poles;

\section{Simulation}

\subsection{Thin-cylinder}

The frequency response data of thin-cylinder with long $1 \mathrm{~m}$ and radius $0.005 \mathrm{~m}$ are calculated by FEKO. The material is ideal conductor, the incident angle is 60 degrees, and the simulation band is $0 \sim 1.5 \mathrm{GHz} .31$ frequency points in the $0.85 \sim 1 \mathrm{GHz}$ are regarded as narrowband data.

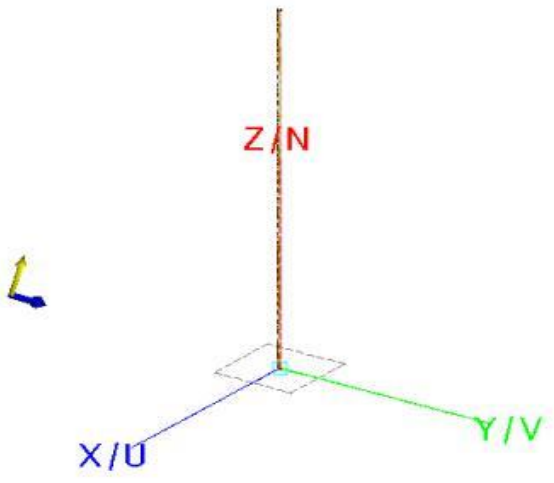

Fig.1. The mesh of thin-cylinder

The error of reconstructed frequency response in different order is shown in the following figure to obtain the appropriate model order.

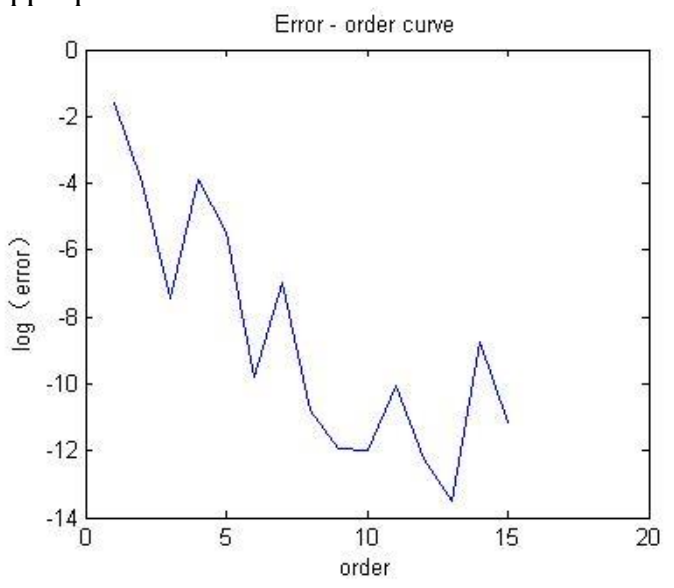

Fig.2. The Error-order curve

As shown in the Fig.2, the chosen order is 13. The reconstructed frequency response data is compared with the actual data. The reconstructed data is basically coincident with the actual data near the sampling narrow band. In other band, the error is very large, but it do not affect the accuracy of poles estimation near the narrowband. 


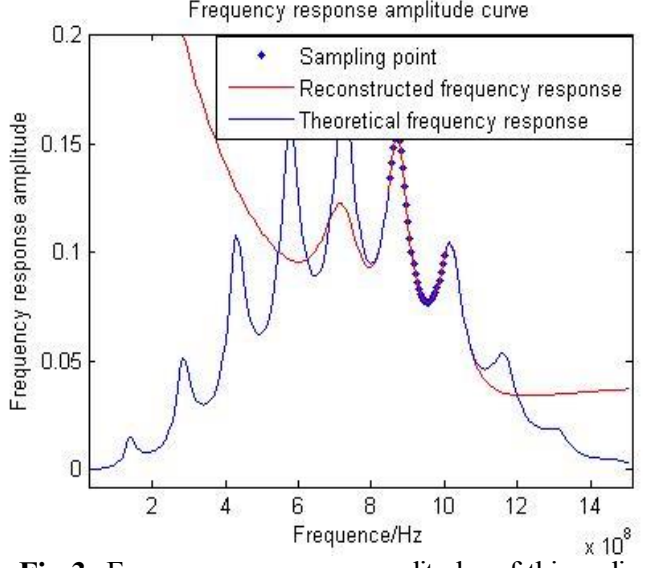

Fig.3. Frequency response amplitude of thin-cylinder

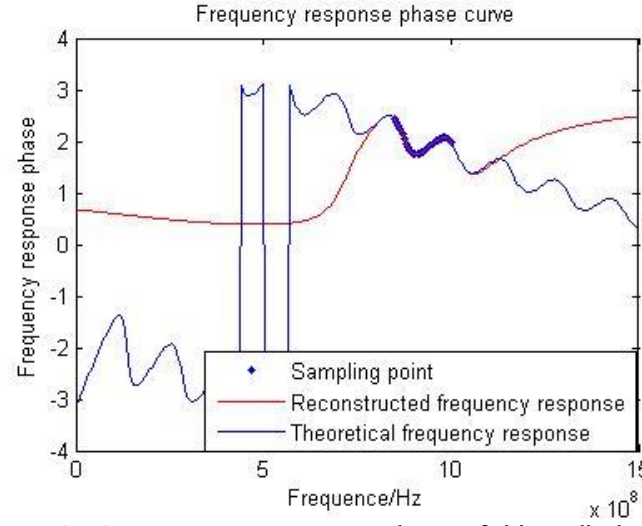

Fig.4. Frequency response phase of thin-cylinder

The poles were computed directly by formula 12 , and the results are as follows:

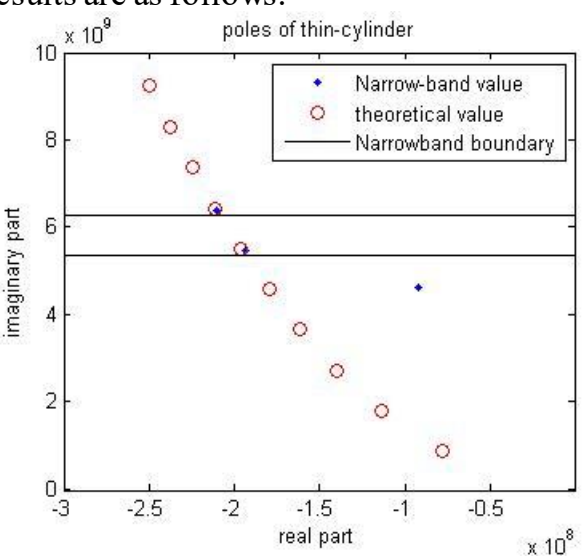

Fig.5. Poles of thin-cylinder

It can be seen that the algorithm extracts both the poles in the sampling narrow band and the poles near the sampling narrow band.

\subsection{Cuboid}

Because the thin-cylinder has only one characteristic length and regular frequency response, the simulation of cuboid is given in this paper. The frequency response data of cuboid with long $0.8 \mathrm{~m}$, width $0.5 \mathrm{~m}$ and high $0.35 \mathrm{~m}$ are calculated by FEKO. The material is ideal conductor and the simulation band is $0 \sim 1 \mathrm{GHz} .21$ frequency points in the $125 \sim 225 \mathrm{MHz}$ are selected as narrowband data.

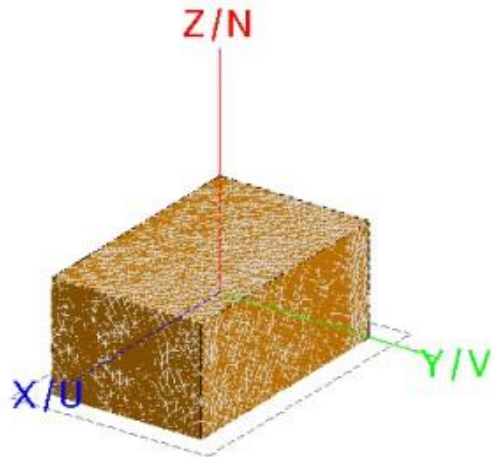

Fig.6. The mesh of cubiod

The poles of the cuboid as Fig.7, and the results are close to the theoretical values.

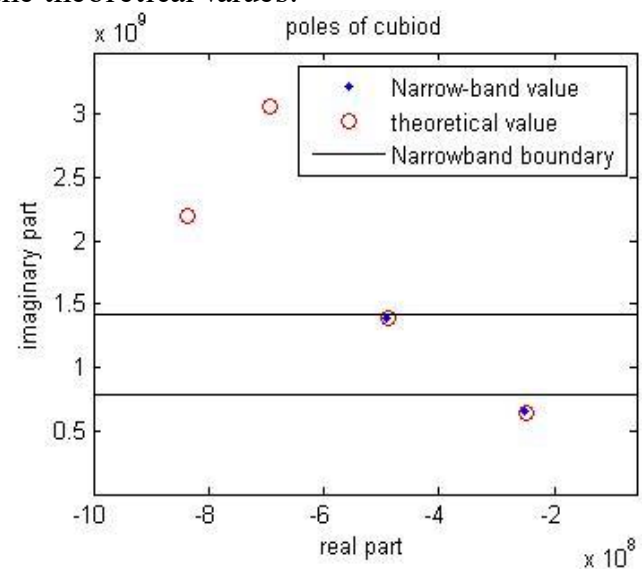

Fig.7. The poles of cubiod

\subsection{Complex target}

A small satellite is taken as an example to verify the effectiveness of the algorithm on complex targets. The observation angle and the mesh is shown in Fig.8. The simulation frequency band is $0 \sim 1 \mathrm{GHz}$.Select two narrowband in $100 \mathrm{MHz} 300 \mathrm{MHz}$ and $500 \mathrm{MHz} 700 \mathrm{MHz}$.

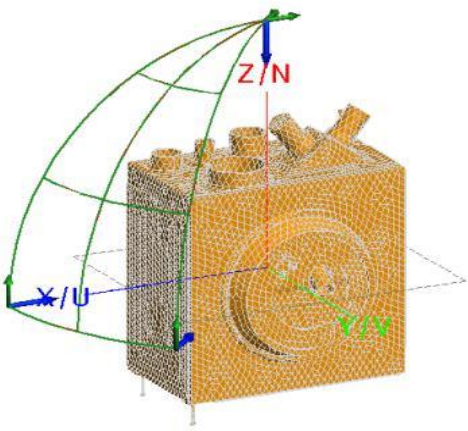

Fig.8. The mesh and observation angles of the satellite

According to the observation angle insensitivity characteristic of poles, the results calculated at different angles should be consistent. The results of this algorithm under three incident angles and the results of wideband data are presented in Fig.7, and the results are consistent. 
The wideband results was calculated by the algorithm of Ref. [9].

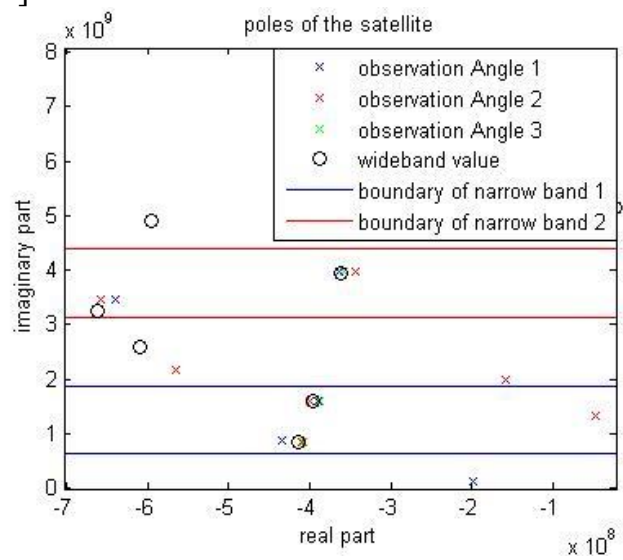

Fig.9. The poles of the satellite

\section{Conclusion}

In this paper, we proposed a poles extraction algorithm which can extract poles from narrow band data. The results show that the method can estimate the poles both in the sampling frequency band and near the sampling frequency band, which means the algorithm has extrapolation ability. It is an effective target recognition method when ultra-wideband data is not available.

\section{Reference}

1. Van Blaricum M, Mittra R. A technique for extracting the poles and residues of a system directly from its transient response, IEEE Transactions on Antennas and Propagation, 23(6).1975, 777-781.

2. Man, Liang, et al. Poles Extracting and Analyzing of Complex Stealth Target Based on Matrix Pencil Method.IEEE International Conference on Computer and Information Technology IEEE Computer Society, (2014)894-898.

3. Choi I S, Lee J H, Kim H T, et al. Natural frequency extraction using late-time evolutionary programming-based CLEAN. IEEE Transactions on Antennas \& Propagation, 51(12), (2003),3285-3292.

4. Baum C E. On the Singularity Expansion Method for the Solution of Electromagnetic Interaction Problems. (1971).

5. Yang Songyan. Research on High Frequency Band Radar Target Features Extraction and Identification. Harbin Institute of Technology,(2016).

6. Jiang Yaowei. Research of semidefinite Programming and its Application. Xidian University,(2009).

7. Xiu Naihua,Luo Ziyan. Semidefinite Programming. Beijing Jiaotong University Press,(2014).

8. K. C. Toh, M. J. Todd, R. H. Tütüncü. SDPT3 - A Matlab software package for semidefinite programming, Version 1.3. Optimization Methods \& Software, 11(1-4),(1999),545-581.
9. Sarkar, T. K., et al. Application of the matrix pencil method for estimating the SEM (singularity expansion method) poles of source-free transient responses from multiple look directions. IEEE Transactions on Antennas \& Propagation 48.4,(2002),612-618. 